\title{
Bioenergy with Carbon Capture and Storage: how carbon storage and biomass resources potentials can impact the development of the BECCS
}

Sandrine Selosse, MINES ParisTech, PSL Research University, CMA - Centre for Applied Mathematics, Rue Claude Daunesse, 06904 Sophia Antipolis, France, sandrine.selosse@mines-paristech.fr

\begin{abstract}
The challenges of climate change involve rethinking the world's energy system. In particular, carbon capture and storage technologies are still presented as a solution to reach ambitious decarbonization targets, and particularly when associated with bioenergy resources due to the negative emission they allow. However, avoiding the required Gt of $\mathrm{CO}_{2}$ emissions by investing in CCS technologies supposes the development of carbon storage capacities and, when associated with bioenergy, a plausible and sustainable potential of biomass resources. This analysis, conducted with the optimization model TIAM-FR (TIMES Integrated Assessment Model, a bottom-up, long-term and multiregional model), highlights the role of these elements in the future development of the BECCS option. More precisely, based, on the one hand, on an advanced methodology of biomass potential assessment, and, on the other hand, on detailed data of storage potential, including onshore and offshore classification, this study shows how such potentials may be a limit to the development of (bioenergy with) carbon capture and storage technologies. This analysis was performed through various scenarios investigated with different levels of potentials and different climate targets on the long-term. As a complement, we also discuss carbon transport costs variation effects and implement a scenario allowing the exclusion of onshore storage due to a hypothetic policy considering public resistance to onshore storage.
\end{abstract}

Keywords: Bioenergy with carbon capture and storage; Carbon storage potential; Biomass potential; Climate scenarios; Long-term modelling, TIAM-FR

\section{Introduction}

Energy and climate are two themes related to the heart of the stakes of societies. The issues of energy supply and more efficient consumption of energy, as well as climate policies, have received increased recognition in recent decades as much in scientific and public debates as political. The challenges of climate change require a complete rethinking of the global energy system and tend to advocate the end of fossil fuels. In particular, the Paris Agreement signed at the end of COP 21 in December 2015, which entered into force in November 2016, is the strong signal of a transition to carbon-free systems by removing our dependence on fossil fuels. Among the main targets, coal was responsible in 2014 for almost $46 \%$ of the world's $\mathrm{CO}_{2}$ emissions and accounted for nearly $41 \%$ of global electricity production, with a contribution of up to $75 \%$ in China, which itself accounts for $42 \%$ of global electricity production by coal [1]. Still present in abundant quantities, coal reserves can ensure a hundred years of consumption; this resource can meet the ever increasing energy needs of many countries, especially those for whom renewable energy still remains too often out of reach. The success of a large-scale energy transition could then involve a more responsible exploitation of these fossil resources. In this context of combating climate change and deploying options for decarbonizing energy systems, carbon capture and storage (CCS) technologies appear particularly interesting. Indeed, these technologies are very largely presented in the results of global long-term planning models as a way to achieve ambitious climate goals by providing clean coal. A further example of this is the proposal for a Directive of the European Parliament and of the Council 2008/0015 on the geological storage of carbon dioxide presented by the Commission in 2008, which well summarizes the situation: "Energy efficiency and renewable energy are the most sustainable long-term solutions for both security of supply and climate protection. However, we will not be able to halve the $\mathrm{CO}_{2}$ emissions of the European Union or the world by 2050 without the possibility of capturing $\mathrm{CO}_{2}$ emitted by 
industrial installations and storing it in geological formations. Internationally, energy consumption from China, India, Brazil, South Africa and Mexico will significantly increase global demand, which will probably have to be met largely thanks to fossil fuels. This legislative framework aims to ensure that $\mathrm{CO}_{2}$ capture and storage technologies are a valid option to reduce emissions and that these technologies are implemented in a safe and responsible manner". For further, introducing CCS to abate emissions increasingly appears incontrovertible to reduce future $\mathrm{CO}_{2}$ emissions but combined with bioenergy to be in line with the limit of a $2{ }^{\circ} \mathrm{C}$ temperature increase.

Bioenergy combined with CCS (BECCS) gained increasing attention since the last decade, as this alternative offers a unique opportunity for net carbon removal from the atmosphere while fulfilling energy needs [2]. The captured $\mathrm{CO}_{2}$ emissions from biomass combustion or conversion are indeed considered as negative insofar as the emissions resulting from the use of biomass are themselves considered as neutral for the system (in because of the assimilation of carbon to atmospheric $\mathrm{CO}_{2}$ via photosynthesis). A growing body of literature assesses the achievement of high $\mathrm{CO}_{2}$ emission reduction targets through the deployment of CCS on an industrial scale and the combination of these technologies with the increasing use of biomass [3, 11]. Indeed, faced with the threat of climate change and tensions over resources (whether energy or water), the growing use of bioenergy is considered a possible track. This is particularly the case as emissions from fossil fuel combustion and cement production have continued to rise during the past decade and that the latest IPCC Assessment Report (AR5) concludes that achieving climate stabilization at safe levels (i.e., below $2{ }^{\circ} \mathrm{C}$ ) will require sustained emission reductions, leading to near-zero or negative emissions (NE) towards the end of this century. In this sense, BECCS is increasingly invoked in integrated assessment models (IAMs), as one option allowing negative emissions, particularly if mitigation is delayed further [12]. For example, in the 2 Degree scenario explored by Muratori et al. [13], CCS significantly contributes to the portfolio of technologies deployed. Indeed, around $17 \mathrm{Gt} \mathrm{CO}_{2} \mathrm{yr}^{-1}$ are stored using CCS technologies in 2050, primarily for electricity and liquid fuels production, with BECCS accounting for $50 \%$ of it. In 2100, about $31 \mathrm{Gt} \mathrm{CO}_{2} \mathrm{yr}^{-1}$ are stored using CCS, with BECCS responsible for 55\%. Furthermore, in the latest IPCC Assessment Report (AR5), 101 of the 116 scenarios with a limited atmospheric concentration at $430-480$ ppm rely on BECCS and about $67 \%$ of these have a BECCS share in primary energy exceeding $20 \%$ in 2100 [14].

However, little is known about the global potential of emerging and future negative emissions technologies, the sustainability and cost of large-scale deployment needed to meet "safe" climate stabilization targets. Furthermore, avoiding the expected gigatonnes of $\mathrm{CO}_{2}$ emissions by investing in CCS technologies implies the development of significant carbon storage capacities. The results of the study published by Viebahn et al. [15] strengthen the interest of this question by showing that "the most crucial pre-condition that must be met is a reliable storage capacity assessment based on sitespecific geological data". In the same manner, the large deployment of BECCS is based on a significant use of biomass resource whose possible level is critical for the plausible development strategies of the sector. The aim of this analysis is to discuss the influence of carbon storage and biomass resources potentials on the possible development of the BECCS technologies.

\section{Modeling approach: The TIAM-FR model}

This analysis is developed with TIAM-FR, the French version of the TIMES Integrated Assessment Model, a widely used, linear programming TIMES family model developed under the IEA's Energy Technology Systems Analysis Program (ETSAP) [16, 17]. More precisely, TIMES is a generator of partial equilibrium techno-economic models representing the energy system of geographical areas and 
time periods determined by the modeler. All TIMES models thus implement a similar mathematical structure, such as for example TIAM, the integrated TIMES model representing the world energy system.

TIAM-FR is therefore a bottom-up energy system model. It depicts the world energy system with a detailed description of different energy forms, technologies and end-uses constituting the Reference Energy System (RES). The RES network links these commodities to several thousand existing and future technologies characterized by their economic and technological parameters in all sectors of the energy system (agriculture, industry, commercial, residential and transport; taking into account conversion and the electricity sector). These technologies can thus be primary sources of commodities (mining technologies or import processes, for example) or processing activities (power plants, refineries, end-use devices, such as cars and heating systems, etc.). The commodities represent energy carriers, energy services, materials, and GHG emissions.

The complete description of each commodity and technology with their associated links must be completed as well as the definition of all the constraints that we want to model: the supply constraints, the technical constraints (the characteristics of each technology), the climate constraints (environmental specifications, policy decisions, etc.) and demand constraints (exogenous demand scenarios). The RES thus provides a representation of the energy chain from primary energy extraction to transformation into final energy uses and to sectors using this final energy, including trade of various energy forms and materials. The Figure 1 shows a synthetic view of the TIAM's energy reference system.

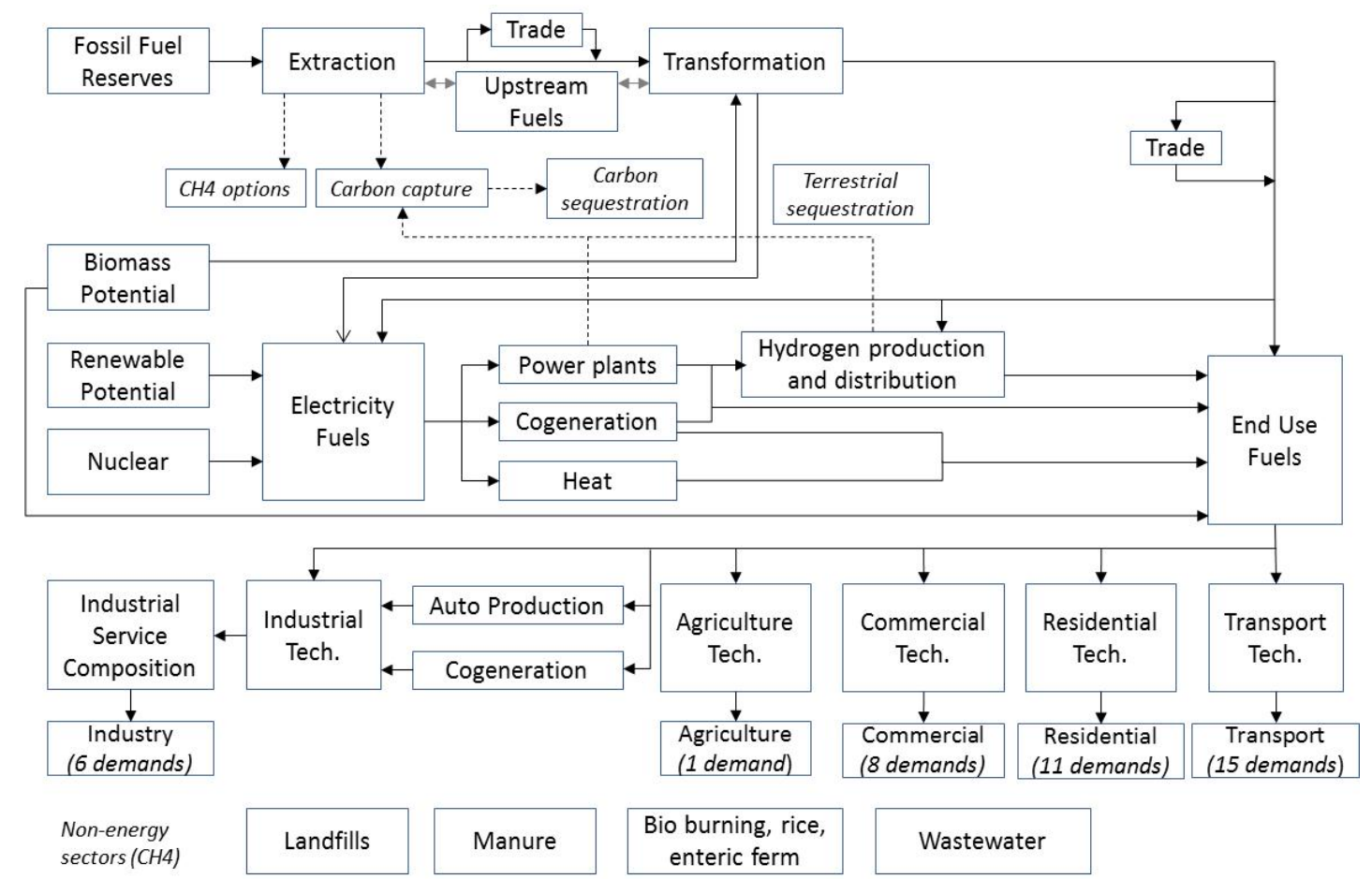

Figure 1: Synthetic view of the TIAM's energy reference system

Thus, the entire energy system is represented, with the objective of satisfying energy service demands at lower cost, under technological and/or environmental constraints posed by the user. One of the interests of this model is its time horizon, which is very long term, until 2100 , to consider global environmental problems such as global warming, which is at the heart of the debate on the 
international scene. TIAM takes into account various greenhouse gases, carbon dioxide $\left(\mathrm{CO}_{2}\right)$, methane $\left(\mathrm{CH}_{4}\right)$ and nitrous oxide $\left(\mathrm{N}_{2} \mathrm{O}\right)$ that the climate module (integrated in the model) allows to consider according to their global warming potential, increasing global temperature, atmospheric GHG concentration and radiative forcing. Driven by end-use demand, the model aims to supply energy services at minimum global cost by simultaneously making decisions on equipment investment and operation, primary energy supply, and energy trade, over this long time period and under a number of environmental and technical constraints.

More precisely as regards the mathematical resolution of the problem, the model minimizes the total discounted cost of the world energy system. It optimizes over the defined time horizon the discounted cost of the energy system under the constraint of satisfaction of the demand. Written in GAMS language, it responds to a problem of minimization linear programming under constraints of the total discounted cost of the energy system, as presented in the Equation 1:

$$
\begin{gathered}
\operatorname{Min} \sum_{i} c_{i} X_{i} \\
\text { sc } \sum_{i} a_{i j} X_{i} \geq b_{j} \text { et } X_{i} \geq 0
\end{gathered}
$$

where, $c_{i}$ represents the coefficients of the objective function; $a_{j i}$ and $b_{j}$, represent the known parameters of the constraints and $X_{i}$, the decision variables.

These decision variables are investments in new capacity (GW), the total installed capacity (GW) and levels of operation or activity (PJ), for each technology, quantities of extracted energy vector, imported or exported (PJ) or the GHG emission levels represented in the model (kt).

It computes the total net present value of the total annual cost of the system. This total annual cost is discounted (here at 5\%) to the selected reference year (here 2010) of the model and includes investment costs, fixed and variable costs of operation and maintenance, costs associated with external resources such as extraction costs, import and export costs, any taxes and subsidies, etc. These updated costs are thus aggregated into a total cost corresponding to the objective function to be minimized by satisfying a certain number of technical and/or environmental constraints, in addition to the constraint of satisfying all the energy service demands. These technical constraints correspond in particular to the respect of the parameters entered for each technology, such as operating limits according to the availability factor, the capacity limits, the lifetime of technologies and the year of availability, etc. As for environmental constraints, they concern, for example, GHG emission limits. So, the objective function comes in the following form (Equation 2):

$$
N P V=\sum_{r=1}^{R} \sum_{y \in \text { years }}\left(1+d_{r, y}\right)^{r e f y-y} * \operatorname{ANN\operatorname {cost}(r,y)}
$$

where $N P V$ is the net present value of the total cost; ANNcost $(r, y)$ is the total annual cost in region $r$ and year $y$; $d_{r, y}$ is the discount rate, refy is the reference year for discounting, years is the set of years and $R$ the set of regions.

The time horizon extends from 2010 to 2100 and the periods within this horizon are determined by the user. It can be divided in terms of years but also in subdivisions in the year. These subdivisions (seasons, day / night) make it possible to take into account certain variable characteristics during the year (electricity consumption, heating demand, solar or hydraulic production, etc.). 
By satisfying the energy service demands implemented at the minimum total cost, decisions are thus made concerning investments, supply of primary and final energies, energy exchanges (etc.) over the defined period and each region of the model. Specifically, the model produces two types of results when computing optimization. First, the primal solution of the linear program provides, for each period of time and region: technology investments; levels of exploitation/operation of technologies; the input/output commodity flows of each technology; the imports/exports of each tradeable commodity; the extraction levels of each primary resource; emissions by technology, by sector and total. In this sense, the structure of the energy system is given as an output.

In addition, the program provides a second solution, called dual solution, which provides the "shadow price" of each commodity of the RES (fuels, materials, energy services, emissions). Thus, information on the marginal costs of environmental measures, such as GHG reduction targets is available. The interest of this type of model is the opportunity they offer to explore the possible energy futures in the long term based on scenarios, i.e. consistent assumptions on the trajectories of the determinants of the system.

As mentioned, TIAM-FR is geographically integrated and representing the world energy system. It offers this representation in 15 regions, as follow: Africa (AFR), Australia-New Zealand (AUS), Canada (CAN), China (includes Hong Kong, excludes Chinese Taipei; CHI), Central and South America (CSA), Eastern Europe (EEU), Former Soviet Union (includes the Baltic states, FSU), India (IND), Japan (JPN), Mexico (MEX), Middle-East (includes Turkey; MEA), Other Developing Asia (includes Chinese Taipei and Pacific Islands; ODA), South Korea (SKO), United States of America (USA) and Western Europe (EU-15, Iceland, Malta, Norway and Switzerland; WEU). These different regions of the world are linked together in terms of energy exchanges.

Finally, emission reduction is achieved through technology and fuel substitutions. Notably, TIAM-FR integrates several carbon capture and sequestration technologies derived from fossil and/or bioenergy resources. The goal of the capture process is to obtain a gas stream with a high concentration of almost pure $\mathrm{CO}_{2}$ at high pressure. There are three modes of capturing $\mathrm{CO}_{2}$ from fossil fuels (coal, oil, natural gas) in the model: 1) post-combustion using a variety of processes such as membrane absorption or separation, 2) pre-combustion with conversion of chemical energy to $\mathrm{H}_{2}$, followed by the simultaneous separation of low-cost carbon and 3) oxy-fuel combustion, characterized by a low cost of $\mathrm{CO}_{2}$ separation, but requiring a supply of $\mathrm{O}_{2}$. For biomass plants, two capture technologies are implemented: pre-combustion capture for the biomass gasification process, and post-combustion capture for direct combustion of biomass. CCS technologies are expected to be available from 2020 onwards. These two types of plants operate only on biomass, which can in this case be solid (wood) or from crops. In the model, biomass is characterized by manifold sources - industrial waste, municipal waste, landfill gas, bioenergy crops, and solid biomass resources.

\section{The future low carbon energy pathways}

To analyze possible development pathways of the investigated future low carbon energy system different long-term UNFCCC $2^{\circ} \mathrm{C}$ objective scenarios under alternative assumptions concerning the available level of carbon storage and the biomass resources potential. Through these sensitivity analyzes, a question arises as to whether the potential of both carbon storage and sustainable biomass is the keystone of the development of the BECCS sector. As a first step, the context of drastic GHG emission reduction by 2050 are considered according to the fact that scenarios in line with the 2 degree objective impose substantial reductions in human GHG emissions by mid-century through large-scale 
changes in energy systems and potentially land use. The range of emissions reductions that allow this goal is considered from 40 to $70 \%$ by 2050 compared to 2010 . This study focuses on the $70 \%$ GHG reduction target. For IPCC, this goal is achievable but it is a significant challenge and face to the fact that GHG emissions keep increasing, the AR5's authors express some doubts as to the realism of such drastic scenarios. So, in a second step, we consider a more long-term climate 2 degree constraint, in other words a limited temperature increase on the time horizon to $2^{\circ} \mathrm{C}$ expressed by a $\mathrm{CO}_{2}$ atmospheric concentration between 441 ppm and 459 ppm by 2100 .

\subsection{Carbon capture and storage: the importance of carbon storage potentials}

The potential for CCS deployment is undoubtedly related to the carbon storage potential, and the supposed high level of this potential in most long-term models does not really appear as a constraint for the development of this decarbonization solution. Since the latter are not always in the same level in the literature [18, 20] or institutional references (US DOE \& NETL [21], Ecofys, NACSA [22], ZEP, etc.), in this context, a state of the art of regional carbon storage potentials was achieved in order to propose new datasets in TIAM-FR and to analyze the sensitivity of CCS investments to different levels of potential. A new distribution between onshore and offshore potential was also added as regards deep saline aquifers and enhanced oil recovery. Many sources were used, as precisely described in Selosse et al. [23], such as for example Wright et al. [24], Bachu [25, 26], Bradshaw et al. [27], Dooley [28], Dahowski et al. [29], Michael et al. [30, 31], Kolenkovi et al. [32], van den Broek et al. [33], and various specific national sources.

Specifically, the new total world storage potential implemented in the model is 10,142 Gt, including 4,907 Gt on onshore deep saline aquifers and 4,117 Gt on offshore deep saline aquifers. For comparison, this level is more optimistic than MERGE (1,466 Gt) and TIMER (5,500 Gt) but is more restrictive than in REMIND, POLES and E3MG, which assume an unlimited potential. Tokimatsu et al. [11], in their analysis of three zero-emission scenarios aimed at achieving the $2{ }^{\circ} \mathrm{C}$ or even lower target, over the period 2010-2150, estimated a geological storage of cumulative $\mathrm{CO}_{2}$ of between about 2,000 and 6,000 Gt. Different storage options exist in the sense that storage capacity data are classified according to regional distribution and storage site type, i.e. deep saline aquifers, coal basins, depleted oil and gas fields, and thus, for all types, according to storage site location, i.e. onshore or offshore (Table 1).

Table 1: Carbon storage by type and by region in TIAM-FR

\begin{tabular}{|c|c|c|c|c|c|c|c|c|c|c|c|c|c|c|c|c|}
\hline Storage site & AFR & AUS & CAN & $\mathrm{CHI}$ & CSA & EEU & FSU & IND & JPN & MEA & MEX & ODA & SKO & USA & WEU & World \\
\hline EOR (Onshore) & 5.2 & 0.0 & 1.7 & 3.8 & 3.9 & 0.6 & 19.1 & 0.4 & 0.0 & 55.3 & 4.6 & 0.0 & 0.0 & 15.4 & 0.0 & 110 \\
\hline EOR (Offshore) & 5.4 & 0.3 & 0.0 & 0.0 & 5.6 & 0.0 & 2.6 & 0.5 & 0.0 & 8.8 & 0.0 & 0.9 & 0.0 & 1.6 & 4.0 & 30 \\
\hline Depl. Oil Fields (Onshore) & 2.6 & 0.0 & 2.5 & 0.8 & 7.5 & 1.5 & 22.5 & 0.0 & 0.0 & 56.3 & 3.8 & 7.5 & 0.0 & 7.5 & 0.4 & 113 \\
\hline Depl. Gas Fields (Onshore) & 17.0 & 0.0 & 12.0 & 7.0 & 29.0 & 5.0 & 202.0 & 0.0 & 0.0 & 31.0 & 0.0 & 8.0 & 0.0 & 33.0 & 0.0 & 344 \\
\hline Depl. Oil Fields (Offshore) & 1.5 & 0.8 & 0.5 & 0.2 & 1.5 & 0.0 & 0.0 & 0.0 & 0.0 & 7.5 & 2.3 & 1.1 & 0.0 & 7.5 & 3.0 & 26 \\
\hline Depl. Gas Fields (Offshore) & 15.0 & 18.0 & 0.0 & 1.0 & 14.0 & 0.0 & 53.0 & 1.0 & 0.0 & 114.0 & 1.0 & 38.0 & 0.0 & 7.0 & 56.0 & 318 \\
\hline Coaldbed Meth. Rec. $<1000 \mathrm{~m}$ & 3.4 & 15.0 & 2.0 & 6.4 & 2.5 & 0.3 & 9.5 & 2.5 & 5.0 & 0.0 & 0.0 & 12.0 & 0.0 & 30.5 & 0.3 & 89 \\
\hline Coaldbed Meth. Rec. $>1000 \mathrm{~m}$ & 3.4 & 15.0 & 2.0 & 6.4 & 2.5 & 0.3 & 9.5 & 2.5 & 5.0 & 0.0 & 0.0 & 12.0 & 0.0 & 30.5 & 0.3 & 89 \\
\hline Deep saline aquifer (Onshore) & 7 & 204 & 22 & 331 & 1545 & 16 & 443 & 32 & 0 & 9 & 50 & 483 & 0 & 1738 & 26 & 4907 \\
\hline Deep saline aquifer (Offshore) & 13 & 475 & 6 & 33 & 455 & 2 & 1657 & 32 & 47 & 1 & 50 & 717 & 0 & 578 & 51 & 4117 \\
\hline TOTAL & 74 & 728 & 49 & 389 & 2067 & 25 & 2418 & 70 & 57 & 283 & 112 & 1279 & 0 & 2449 & 142 & 10142 \\
\hline
\end{tabular}

In this study, a sensitivity analysis was carried out considering lower assumptions about regional carbon storage potential, or more specifically considering Hendricks’ assumptions: 
- Best scenario with a total world storage potential of 1,706 Gt, including $609 \mathrm{Gt}$ on onshore depleted gas fields and 302 Gt on offshore depleted gas fields (Table 2).

Table 2: Carbon storage by type and by region in Hendricks [18] - Best scenario

\begin{tabular}{|c|c|c|c|c|c|c|c|c|c|c|c|c|c|c|c|c|}
\hline Storage site & AFR & AUS & CAN & $\mathrm{CHI}$ & CSA & EEU & FSU & IND & JPN & MEA & MEX & ODA & SKO & USA & WEU & World \\
\hline EOR (Onshore) & 6.2 & 0.0 & 0.4 & 3.0 & 10.4 & 0.9 & 21.8 & 0.4 & 0.0 & 62.0 & 0.0 & 1.0 & 0.0 & 6.2 & 0.1 & 112 \\
\hline EOR (Offshore) & 8.1 & 0.5 & 0.3 & 0.5 & 8.6 & 0.0 & 2.9 & 0.6 & 0.0 & 9.3 & 0.0 & 1.4 & 0.0 & 0.5 & 4.0 & 37 \\
\hline Depl. Oil Fields (Onshore) & 2.6 & 0.0 & 2.5 & 0.8 & 7.5 & 1.5 & 22.5 & 0.0 & 0.0 & 56.3 & 3.8 & 7.5 & 0.0 & 7.5 & 0.4 & 113 \\
\hline Depl. Gas Fields (Onshore) & 37.6 & 0.3 & 14.7 & 11.7 & 28.3 & 6.8 & 197.3 & 13.4 & 0.0 & 260.4 & 0.0 & 9.8 & 0.0 & 13.7 & 15.4 & 609 \\
\hline Depl. Oil Fields (Offshore) & 1.5 & 0.8 & 0.5 & 0.2 & 1.5 & 0.0 & 0.0 & 0.0 & 0.0 & 7.5 & 2.3 & 1.1 & 0.0 & 7.5 & 3.0 & 26 \\
\hline Depl. Gas Fields (Offshore) & 17.8 & 17.3 & 0.8 & 0.4 & 25.0 & 0.0 & 73.5 & 5.2 & 0.0 & 85.7 & 0.0 & 34.9 & 0.0 & 2.1 & 38.8 & 302 \\
\hline Coaldbed Meth. Rec. $<1000 \mathrm{~m}$ & 3.8 & 5.7 & 4.3 & 79.0 & 1.0 & 0.4 & 12.5 & 1.0 & 0.1 & 0.0 & 0.0 & 9.5 & 0.0 & 15.9 & 0.5 & 133 \\
\hline Coaldbed Meth. Rec. $>1000 \mathrm{~m}$ & 3.8 & 5.7 & 4.3 & 79.0 & 1.0 & 0.4 & 12.5 & 1.0 & 0.1 & 0.0 & 0.0 & 9.5 & 0.0 & 15.9 & 0.5 & 133 \\
\hline Deep saline aquifer (Onshore) & 16.2 & 8.5 & 13.9 & 12.2 & 23.4 & 3.1 & 7.0 & 10.6 & 0.0 & 9.1 & 0.0 & 2.6 & 0.0 & 13.0 & 3.5 & 123 \\
\hline Deep saline aquifer (Offshore) & 31.8 & 19.6 & 3.4 & 1.2 & 6.9 & 0.3 & 26.0 & 10.6 & 1.9 & 0.6 & 0.0 & 3.8 & 0.0 & 4.3 & 6.8 & 117 \\
\hline TOTAL & 129 & 58 & 45 & 188 & 114 & 13 & 376 & 43 & 2 & 491 & 6 & 81 & 0 & 87 & 73 & 1706 \\
\hline
\end{tabular}

- Low scenario with a total storage potential of $572 \mathrm{Gt}$, including $113 \mathrm{Gt}$ on onshore depleted oil fields, $223 \mathrm{Gt}$ on onshore depleted gas fields and $169 \mathrm{Gt}$ on offshore depleted gas fields (Table 3).

Table 3: Carbon storage by type and by region in Hendricks [18] - Low scenario

\begin{tabular}{|c|c|c|c|c|c|c|c|c|c|c|c|c|c|c|c|c|}
\hline Storage site & AFR & AUS & CAN & $\mathrm{CHI}$ & CSA & EEU & FSU & IND & JPN & MEA & MEX & ODA & SKO & USA & WEU & World \\
\hline EOR (Onshore) & 0.5 & 0.0 & 0.0 & 0.2 & 0.8 & 0.1 & 1.7 & 0.0 & 0.0 & 5.1 & 0.0 & 0.1 & 0.0 & 0.8 & 0.0 & 9 \\
\hline EOR (Offshore) & 0.5 & 0.0 & 0.0 & 0.0 & 0.5 & 0.0 & 0.2 & 0.1 & 0.0 & 0.8 & 0.0 & 0.1 & 0.0 & 0.1 & 0.3 & 3 \\
\hline Depl. Oil Fields (Onshore) & 2.6 & 0.0 & 2.5 & 0.8 & 7.5 & 1.5 & 22.5 & 0.0 & 0.0 & 56.3 & 3.8 & 7.5 & 0.0 & 7.5 & 0.4 & 113 \\
\hline Depl. Gas Fields (Onshore) & 15.2 & 0.1 & 6.7 & 4.0 & 9.9 & 2.9 & 71.3 & 4.1 & 0.0 & 92.6 & 0.0 & 2.9 & 0.0 & 7.8 & 5.0 & 223 \\
\hline Depl. Oil Fields (Offshore) & 1.5 & 0.8 & 0.5 & 0.2 & 1.5 & 0.0 & 0.0 & 0.0 & 0.0 & 7.5 & 2.3 & 1.1 & 0.0 & 7.5 & 3.0 & 26 \\
\hline Depl. Gas Fields (Offshore) & 7.4 & 7.2 & 0.7 & 0.3 & 10.9 & 0.0 & 26.1 & 1.9 & 0.0 & 70.6 & 0.0 & 19.1 & 0.0 & 1.9 & 23.2 & 169 \\
\hline Coaldbed Meth. Rec. $<1000 \mathrm{~m}$ & 0.0 & 0.0 & 0.0 & 0.0 & 0.0 & 0.0 & 0.0 & 0.0 & 0.0 & 0.0 & 0.0 & 0.0 & 0.0 & 0.0 & 0.0 & 0 \\
\hline Coaldbed Meth. Rec. >1000m & 0.0 & 0.0 & 0.0 & 0.0 & 0.0 & 0.0 & 0.0 & 0.0 & 0.0 & 0.0 & 0.0 & 0.0 & 0.0 & 0.0 & 0.0 & 0 \\
\hline Deep saline aquifer (Onshore) & 2.1 & 1.1 & 1.8 & 1.5 & 2.9 & 0.4 & 0.9 & 1.4 & 0.0 & 1.1 & 0.0 & 0.3 & 0.0 & 1.7 & 0.4 & 15 \\
\hline Deep saline aquifer (Offshore) & 4.0 & 2.4 & 0.4 & 0.2 & 0.9 & 0.0 & 3.2 & 1.4 & 0.2 & 0.1 & 0.0 & 0.5 & 0.0 & 0.5 & 0.9 & 15 \\
\hline TOTAL & 34 & 12 & 13 & 7 & 35 & 5 & 126 & 9 & 0 & 234 & 6 & 32 & 0 & 28 & 33 & 572 \\
\hline
\end{tabular}

\subsection{An advanced methodology of biomass potential assessment}

With growing concerns about climate change, countries are increasing efforts to reduce dependency on fossil energy sources, the major source of $\mathrm{CO}_{2}$, by replacing them with cleaner energy sources including bioenergy. As regards biomass resources, this is true only in the case of sustainable management of biomass. Recent assessments of the potential of the biomass energy resource point to the fact that this resource could, in a sustainable way, contribute 160 to $270 \mathrm{EJ}$ (exajoules) to the world's primary energy supply in 2050 [34]. Nevertheless, a number of obstacles must be overcome before such potential can be exploited, one of the main ones being that the most important biomass resources are generally not located close to urban and industrial areas. In addition, countries that could become important biomass producers are not systematically the same as those that could become important users. In parallel, while international bioenergy markets are still in their infancy, the international trade in biofuels, wood pellets, ethanol and palm oil is expanding. In addition, the evolution of thermal pretreatment technologies, such as roasting, gasification and pyrolysis, stimulate 
the production of bioenergy products, the latter being able to be transported over long distances (because they are very homogeneous, having a high density energy and not sensitive to biodegradation). The development of coal / gas and biomass co-firing plants increases the range of end-use applications for biomass, such as the potential development of the BECCS. However, the global impact that increased exchanges of these resources could have on the development of bioenergies and energy systems is not yet very clear. The integrated models used to estimate the development of the global energy system generally do not have sufficient variations and data for future growth in biomass supply and heat pre-heat technologies, for example. They are also generally not taken into account (as was initially the case in the TIAM model). In the literature, the biomass potential varies considerably depending on the different assumptions about land use, yield development, food consumption and other sustainability criteria, such as water scarcity and biodiversity loss [10][35]. Studies have shown that the greatest potential for biomass production will occur in areas with favorable climatic conditions and abundant land resources, such as Latin America and North America, Central Asia and the Pacific, China and sub-Saharan Africa. On the other hand, the main demands come from OECD countries and South-East Asia [34][36]. In long-term prospective, it is important to carefully examine the model results. After studying carbon storage potentials, it was important to deepen the discussion of potential biomass resources that are expected in the case of investments in BECCS. Then, taking into account this point, the evaluation of the potential of the biomass resource and the implementation of international biomass exchanges and pretreatment technologies in TIAM-FR were key development elements to evaluate the possible role of BECCS. A lot of development work had been done on the representation of the resources themselves and their potential, which has been endogenized in the model according to the evolution of bioenergy needs but also and above all taking into account considerations related to land use, surface area, yield and uses for food, construction, etc. [37, 40]. This work made it possible to refine the consideration of biomass, which can be decisive with regard to the place occupied by biomass-associated with carbon capture and storage (BECCS) in climate scenarios.

More precisely, according to the work of Kang [38], in order to make it possible to choose between several energy crops according to the corresponding uses and to introduce the competition between the resources, the potential of the energy crops is estimated at the level of the available surface area for each of the fifteen regions of the TIAM-FR model. For this estimate, food security is prioritized to avoid conflict with food production around the world. The implementation of this food-first approach is based on Smeets et al. [41]. An approach of optimization of the land use is also applied. Based on the projection of domestic food production, agricultural residues are thus estimated taking into account the environmental criterion. Secondly, the resources available in forest biomass are estimated by limiting themselves to sustainable growth under a conservative constraint. A sustainability condition is assumed in order to limit the exploitation of the land area, i.e. pasture, grass and other wooded land according to the classification of GAEZ, the Global System of Agro-Ecological Zones [42] and FAO. The forest is thus conserved as much as possible over the entire time horizon. Finally, forest residues are also estimated by applying environmental criteria to avoid biological degradation of land. The GAEZ projection of agricultural yields was selected: first the agro-climatic productivities and to supplement, if need be, when the projection was not available, the agro-ecological productivities. Another challenge identified to estimate the area for food production is the limit of use of the irrigation system. Agricultural yields differ according to the water supply system used. Despite higher productivities with the irrigation system than with the rainfed system, its deployment must be limited in the light of several constraints such as available water or economic and ecological aspects. The 
potential of the irrigation system proposed by AQUASTAT [43] is applied for estimate the area requested. Moreover, the assessment of the potential for agricultural residues differs according to the source of the resources, i.e. whether they are primary residues, which are generated at the time of harvest, or secondary residues, which are by-products of the transformation of cultures into final product. As a first step, the potential for primary agricultural residues is estimated based on the RPR, the Residue to Product Ratio and the recovery factor. The data of RPR and rate of production of residues from unit production per crop mainly come from [44, 45] and were supplemented by data from SERI, the Sustainable Europe Research Institute [46] for missing elements. Finally, crop residues at harvest are assessed by multiplying the amount of domestic production of each crop by the corresponding RPR and the recovery rate, as described follow (Equation 3):

$$
\operatorname{Res}_{c, r, t}=\text { Dom }_{c, r, t} \times T_{c, r}^{R P R} \times T_{r}^{\text {Recovery }}, \forall c \in \text { Cultures, } \forall r \in \text { Country/regions, } \forall t \in \text { Year }
$$

where $\operatorname{Res}_{c, r, t}$ is the quantity of available residues from culture $c$, in region $r$, in year $t$; $\operatorname{Dom}_{c, r, t} \mathrm{~s}$ the quantity of the production of culture $c$, in region $r$, in year $t ; T_{c, r}^{R P R}$ is the Residue to Product Ratio of culture $c$, in region $r ; T_{r}^{\text {Recovery }}$ is the residue recovery factor in region $r$.

Several studies on the sustainable use of agricultural residues conclude that between $25 \%$ and $50 \%$ of agricultural residues from crops can be recovered without impacting land performance and 5\% for vegetables and fruit $[47,51]$. A recovery rate of $25 \%$ is assumed for general crops and $5 \%$ for vegetables and fruits. Based on this methodology, the agricultural biomass potential was estimated for each of the 15 regions of the TIAM-FR model. These results highlight the importance of technical progress in the agricultural sector to be able to provide more biomass in the world with less impact of land use change. Thus, the area available for bioenergy production is well informed in the model and is linked to processes of conversion to energy, the choice of commodities then being endogenous to the model. The potential of energy crops can therefore vary according to several criteria: the technoeconomic parameters of the bioenergy sector according to regions and periods, the availability of technologies, etc. Nevertheless, in order to position the possible extent of our available potential, the energy potential is estimated assuming full land use for the production of lignocellulosic crops. As a result the energy crop potential can be estimated at $9 \mathrm{EJ}$ in the BIO_LO scenario (the most restrictive), at 154 EJ in the BIO_MID scenario (the most likely, but achievable) and at 267 EJ in the BIO_HI scenario (the most optimistic) in 2050.

As regards the evaluation of the supply of wood resources, it is limited to the sustainable surplus of forest supply by separately estimating wood supply and demand. In the case of supply, three different sources were estimated: trees in the forest, other wooded lands and finally, trees outside the forest (TOF). Regarding the demand for wood, the consumption of industrial round wood is estimated. As for agricultural residues, forest residues are calculated for both primary residues that occur during cutting and for secondary residues that are generated during wood processing. As a first step, the primary residues are calculated on the basis of the quantities of industrial round wood removed and the rate of generation of residues which is of the order of $60 \%$. Regarding the recovery rate, a rate of $25 \%$ is assumed, in line with the rate applied for primary agricultural residues in a conservative context. Then, to estimate the secondary residues, the quantities of industrial round wood consumed is used, a generation rate of $50 \%$ and a recovery rate of $50 \%$. In both cases, primary and secondary residues, a 
dry matter ${ }^{1}$ conversion rate of $0.56 \mathrm{t} \mathrm{DM}^{\mathrm{m}} / \mathrm{m}^{3}$ is applied (which is the default value considered by the IPCC) and an energy content of $18.3 \mathrm{MJ} / \mathrm{kg}$. This estimation method is extracted from the literature [41][50][52]. Thus, at the end, the global wood supply potential, in line with these principles of sustainability, is estimated at $61.2 \mathrm{EJ} / \mathrm{year}$ for the economic potential and $107.5 \mathrm{EJ} / \mathrm{year}$ for the technical potential in 2050. In this study, the economic potential is assumed in order to analyze the future deployment of bioenergy, disaggregated by resource type: wood, cutting residues and processing residues.

\section{Results}

The impact of carbon storage and biomass potentials on the deployment of BECCS technologies is discussed based on these alternative climate and resources context. The considered scenarios are presented in the Table 4, the scenario with mid-low potential of biomass and high storage capacities being here considered as the standard scenario to face the climate constraint.

Table 4: Alternative low carbon pathways

\begin{tabular}{|c|c|c|c|c|}
\hline Scenario & Targeted year & Climate constraint & Carbon storage & Biomass 2050 potential \\
\hline 2050-70-ccsHi-BioMid & \multirow{9}{*}{2050} & \multirow{9}{*}{ 70\% GHG mitigation } & \multirow{3}{*}{$10,142 \mathrm{Gt}$} & $215 \mathrm{EJ}$ \\
\hline 2050-70-ccsHi-BioHi & & & & $328 \mathrm{EJ}$ \\
\hline 2050-70-ccsHi-BioLo & & & & $70 \mathrm{EJ}$ \\
\hline 2050-70-ccsMid-BioMid & & & \multirow{3}{*}{$1,706 \mathrm{Gt}$} & $215 \mathrm{EJ}$ \\
\hline 2050-70-ccsMid-BioHi & & & & $328 \mathrm{EJ}$ \\
\hline 2050-70-ccsMid-BioLo & & & & $70 \mathrm{EJ}$ \\
\hline 2050-70-ccsLo-BioMid & & & \multirow{3}{*}{$572 \mathrm{Gt}$} & $215 \mathrm{EJ}$ \\
\hline 2050-70-ccsLo-BioHi & & & & $328 \mathrm{EJ}$ \\
\hline 2050-70-ccsLo-BioLo & & & & $70 \mathrm{EJ}$ \\
\hline 2100-2D-ccsHi-BioMid & \multirow{9}{*}{2100} & \multirow{9}{*}{$2^{\circ} \mathrm{C}$ temperature increase limit } & \multirow{3}{*}{$10,142 \mathrm{Gt}$} & $215 \mathrm{EJ}$ \\
\hline 2100-2D-ccsHi-BioHi & & & & $328 \mathrm{EJ}$ \\
\hline 2100-2D-ccsHi-BioLo & & & & $70 \mathrm{EJ}$ \\
\hline 2100-2D-ccsMid-BioMid & & & \multirow{3}{*}{$1,706 \mathrm{Gt}$} & $215 \mathrm{EJ}$ \\
\hline 2100-2D-ccsMid-BioHi & & & & $328 \mathrm{EJ}$ \\
\hline 2100-2D-ccsMid-BioLo & & & & $70 \mathrm{EJ}$ \\
\hline 2100-2D-ccsLo-BioMid & & & \multirow{3}{*}{$572 \mathrm{Gt}$} & $215 \mathrm{EJ}$ \\
\hline 2100-2D-ccsLo-BioMHi & & & & $328 \mathrm{EJ}$ \\
\hline 2100-2D-ccsLo-BioLo & & & & $70 \mathrm{EJ}$ \\
\hline
\end{tabular}

First (in our "base" 2050-70-ccsHi-BioMid scenario), results show that the model responds to the $70 \%$ GHG mitigation target by 2050 by a large-scale deployment of CCS technologies in the electricity sector, first installed technologies starting at 2020. Thus, 39\% of the electricity produced in 2050 comes from power plants equipped with capture technologies. At the global level, $55.9 \%$ of the deployed CCS plants operate with biomass. For the $44.1 \%$ operating with fossil fuels, there is a preponderance of coal plants, some of them being co-firing with biomass (limited to $20 \%$ of biomass resources). Excluding co-firing, first BECCS technologies investments occur in 2030 but bioplants with CCS start particularly to be deployed from 2040 representing 38\% of CCS investments. In this scenario, in terms of biomass consumption, in 2020, 74.9\% of resources used by CCS plants are coal, the rest being biomass. Due to the drastic climate constraint, by 2020, CCS technologies are deployed in coal power plants and combined with a minimal use of biomass. Coal-biomass proportions progressively reversed during the time period, until 2050 when biomass represents $68 \%$ of the

\footnotetext{
${ }^{1}$ Dry matter (DM) is that which remains after redrying at $105^{\circ} \mathrm{C}$ for $15 \mathrm{~h}$ a previously dried and ground sample.
} 
resources used in plants equipped with CCS. In this scenario, the development of carbon capture technologies in the electricity sector allows storing more than $13 \mathrm{Gt}$ of $\mathrm{CO}_{2}$ in 2050, 2/3 can be considered as negative emissions because of the combustion of biomass resources. In 2030, $4 \mathrm{Gt}$ of $\mathrm{CO}_{2}$ can be stored due to CCS investments, essentially coming from fossil fuels combustion (70\%). Investments in CCS technologies also occur in other sector of the energy system. Particularly, in the transport sector, the CCS installation in second generation of cellulose ethanol plants and in the biodiesel processing induces the storage of $2.8 \mathrm{Gt}$ of $\mathrm{CO}_{2}$ in 2050, considered as negative emissions. First installations occur in 2030. From 2040, some hydrogen production processes start to be equipped with CCS technologies and lead to the storage of $340 \mathrm{Mt}$ of $\mathrm{CO}_{2}$, reaching $615 \mathrm{Mt}$ in 2050.

Alternative contexts in terms of carbon storage and biomass potentials impact more or less the development of BECCS. Indeed, as firstly regards the electricity sector, it is interesting to note that a lower level of $\mathrm{CO}_{2}$ storage capacity, as assumed in the medium level of 1,706 Gt (against 10,142 Gt), does not induce a decrease in investments in CCS technologies. This is not the case of the low assumption of carbon storage potential where a decrease of around 12 points of percentage occurs when the available capacity of storage is low, i.e. $572 \mathrm{Gt}$, whatever the level of biomass potential. On the other hand, Table 5 highlights that the impact is more significant with regard the BECCS development more precisely.

Table 5: Share of CCS in the world production of electricity in 2050

\begin{tabular}{|c|c|c|c|c|}
\hline \multicolumn{2}{|c|}{$\begin{array}{c}\text { Ambitious climate scenario - } \\
70 \% \text { GHG mitigation target }\end{array}$} & High & Medium & Low \\
\cline { 2 - 4 } & High & $45 \%$ (BECCS: 70\%) & $39 \%$ (BECCS: $55.9 \%)$ & $27 \%$ (BECCS: $18.1 \%)$ \\
\cline { 2 - 5 } $\begin{array}{c}\text { Carbon storage } \\
\text { potential }\end{array}$ & Medium & $45 \%$ (BECCS: $69.8 \%)$ & $39 \%$ (BECCS: $56.3 \%)$ & $27 \%$ (BECCS: $18.2 \%)$ \\
\cline { 2 - 5 } & Low & $33 \%$ (BECCS: $93.9 \%)$ & $28 \%$ (BECCS: $76.7 \%)$ & $15 \%$ (BECCS: $33.5 \%)$ \\
\hline
\end{tabular}

The lower is the carbon storage potential; the higher is the share of BECCS in the CCS development. More precisely, in the medium biomass potential case, BECCS thus increases to $76.7 \%$ of the CCS investment against $56 \%$ in the higher carbon storage potential cases. This can be explained by the fact that, in 2050, the development of CCS is not limited by the potential of carbon storage when the latter is assumed to be high and medium. However, when the potential of carbon storage is low, as assumed in the low case, not only CCS investments are limited (representing 28\% against $39 \%$ in the biomass base/medium case) but BECCS is privileged as possible in order to benefit from negative emissions, allowing to satisfy the drastic climate constraint. This higher level of development of BECCS (compared with fossil CCS) when the carbon storage potential is lowest, occurs in all cases, whatever the level of biomass potential. Indeed, in the highest case of biomass potential, the CCS development decreases from $45 \%$ in high and medium case of carbon storage, to 33\% in the low case of carbon storage, but the share of BECCS increases from 70\% to 93.9\%. In the same way, in the lowest case of biomass potential, the CCS development decreases from 27\% in high and medium case of carbon storage, to $15 \%$ in the low case of carbon storage but the share of BECCS increases from $18.2 \%$ to $33.5 \%$. However, we can note that the impact of biomass potential is more significant on the development of both CCS and BECCS. In case of lower potential of biomass, CCS technologies represent between 27 and 15\% of the low carbon power generation against between 45 and 33\% in case of highest level of biomass resources.

In terms of $\mathrm{CO}_{2}$ sequestrated, according to the potential of carbon storage and of biomass resources, the development of CCS technologies in the power sector prevents from $15.8 \mathrm{Gt}$ to $4.4 \mathrm{Gt}$ of $\mathrm{CO}_{2}$ 
from going into the atmosphere, including from $12 \mathrm{Gt}$ to $2.2 \mathrm{Gt}$ of negative emissions (Table 6). It is interesting to note that the effect of carbon storage and biomass resources potentials on the development of BECCS is similar in other sector of the energy system.

Table 6: Gt of negative emissions ( $\mathrm{CO}_{2}$ sequestrated in 2050 from $\left.\mathrm{BECCS}\right)$

\begin{tabular}{|c|c|c|c|c|}
\hline \multirow{2}{*}{$\begin{array}{c}\text { Ambitious climate scenario - } \\
70 \% \text { GHG mitigation target }\end{array}$} & \multicolumn{3}{|c|}{ Biomass potential } \\
\cline { 2 - 5 } & High & $12 \mathrm{Gt}$ & Medium & Low \\
\hline \multirow{2}{*}{$\begin{array}{c}\text { Carbon storage } \\
\text { potential }\end{array}$} & Medium & $12 \mathrm{Gt}$ & $8.9 \mathrm{Gt}$ & $2.8 \mathrm{Gt}$ \\
\cline { 2 - 5 } & Low & $11 \mathrm{Gt}$ & $7.7 \mathrm{Gt}$ & $2.8 \mathrm{Gt}$ \\
\cline { 2 - 5 } & \multicolumn{2}{|c}{} & \\
\hline
\end{tabular}

As previously mentioned, a 70\% reduction of GHG emissions by the middle of the century constitutes an ambitious challenge to reach in order to stay in line with the $2{ }^{\circ} \mathrm{C}$ objective consensually committed by all countries. Then we implemented another climate constraint, always in line with the $2{ }^{\circ} \mathrm{C}$ objective but with a more long-term target and with, as it were, the highest challenge deferred to the second half of the century. The time horizon is now 2100 and the energy system can consider more time to decarbonize and deploy alternative low-carbon solutions. In the same way, the carbon storage potential has to be taken into account from 2010 to 2100 (unlike the previous situation where the time period was from 2010 to 2050). Indeed, in this climate scenario where the same alternative potentials (as regards carbon storage and biomass resources) were assumed, the electricity generation by power plant with CCS represent between $1 \%$ and $11 \%$ according to the scenario. CCS investments only start occurring by 2060, earlier renewables investments being then privileged. In this case, low carbon storage potential involves, whatever the biomass potential, a drastically lower level of development of CCS technologies. This lower level of CCS development is accentuated by a lower level of biomass potential. On the other hand, in case of higher level of carbon storage, it is interested to note that lower level of biomass potential involved an increase of the CCS deployment. This time, CCS based on fossil resources (or coal and biomass co-firing) is privileged by comparison with BECCS. The development of BECCS in the electricity sector is favored when biomass potential is higher. Between 81 and 92\% of CCS in this case then represent BECCS options. In this case, it seems that CCS is more here considered as a complementary solution. In this context, it is interesting to note than CCS from other sectors than electricity one is more developed than in the previous situation. At the end, between 5 and $15.3 \mathrm{Gt}$ of $\mathrm{CO}_{2}$ have to be stored in 2100 due to the development of CCS technologies in order to satisfy the climate constraint. The stored $\mathrm{CO}_{2}$ essentially constitutes negative emissions, as they come from biomass sources, except when low biomass resources potential is assumed. In this case, captured $\mathrm{CO}_{2}$ comes from fossil sources. Also, when lowest carbon storage potential is assumed, around $5 \mathrm{Gt}$ of $\mathrm{CO}_{2}$ has to be stored, but it reaches $7 \mathrm{Gt}$ when highest biomass potential is assumed. In all cases, $\mathrm{CO}_{2}$ emissions come from biomass resources. Indeed, in this case, benefit from negative emissions is particularly favored.

\section{Conclusion}

As previously stated, among the low-carbon technology options, carbon capture and storage technologies are widely presented as a solution for achieving ambitious climate goals, particularly when associated with biomass. The challenge of the Paris Agreement is to support countries in this transition to a new low-carbon world while there is still a significant gap between the countries' ambitions expressed in their commitments and the ultimate goal of maintaining the rise temperatures at $+2{ }^{\circ} \mathrm{C}$. It can be described as the most successful global diplomacy in the fight against climate 
change through the institutionalization of a new paradigm that it engenders [53]. To further mitigate GHG emissions, a key measure of success is how far and how fast the Paris Agreement will encourage more ambitious actions [54]. Ambitious and realistic goals must therefore be discussed in the light of realistic technological change and the availability of technological solutions in all regions of the world. These discussions include long-term solutions, such as the development of CCS technologies or renewable energies, in response to a constraint that affects the energy mix. As these results highlight, in case of ambitious and drastic climate target, by the mid-century, CCS technologies appears as a valuable options and they are particularly favored. But these solutions, notably when concerns the possibility of negative emissions with BECCS, are significantly dependent on biomass resources potentials; more than on the carbon storage potentials, except in the case of a very low level of storage capacity. Interestingly, when biomass resources are limited, BECCS options are favored by comparison with (fossil) CCS, in order to increase the possible negative emissions quantities.

The aim of this research is to highlight the challenges posed by this technological development, which is also a critical factor for the future international climate regime. Previous results highlight that the response to carbon constraints is largely based on investments in CCS technologies. However, the feasibility of avoiding so much Gt of $\mathrm{CO}_{2}$ by investing in these technologies is still debatable and will strongly depend on the condition of development of biomass resources insofar as the more sustainable and conservative are the constraints, the more limited is the biomass resources potential. Al the challenge is so to maintain a beneficial balance in fine. As regards carbon storage potential, it seems to be sufficient to satisfy the climate constraint developing CCS technologies, but it will need to go beyond acceptability issues, the consideration of which would lead to a significant reduction in the level of carbon storage potential, and thereby, as evidenced by these results, the deployment of CCS and BECCS.

Deploying these technologies at this scale for mitigation purposes therefore requires the implementation of incentive and regulation policies, this concerns as well as a regulatory framework to support the CCS business models (Rai et al., 2010) than the societal and environmental implications of the development of these options. More precisely, this implies that governments play a decisive role in BECCS technologies. The IEA, in its 2016 edition of the Energy Technology Outlook (ETP), states that moderate progress had been made in 2015 in the area of CCS and that both industry and governments will have to invest significantly in projects and technology development to ensure that CCS achieves the expected goal of annual $\mathrm{CO}_{2}$ storage (IEA, 2016). Significant development of CCS will also imply that storage sites are socially acceptable. It will also be critical to take into account that the benefits of BECCS negative emissions are only effective in the case of sustainable use of biomass. In this area, the restoration and preservation of forests is another key factor, just as so many other challenges remain to be overcome. So, given the influence that carbon storage and biomass resources potentials play, and, in turn, the role played by states in these areas, it seems important that the official guidelines and positioning to be followed are previously and quickly stated, which would allow a better visibility and anticipation of the role that BECCS can play in the future.

\section{Acknowledgements}

This research was supported by the Chair Modeling for sustainable development, driven by MINES ParisTech, Ecole des Ponts ParisTech, and AgroParisTech, supported by ADEME, EDF, GRTgaz, SCHNEIDER ELECTRIC and the French Ministry of Ecology, Sustainable Development and Energy. 
The authors thank Olivia Ricci from the Centre for Economics and Management in Indian Ocean (CEMOI) of the University of Reunion Island for her contribution on the potential of carbon sequestration, and Seungwoo Kang from IRENA for his work on the biomass potential during his doctoral studies at the MINES ParisTech Center for Applied Mathematics (CMA) at Sophia Antipolis, France.

\section{References}

[1] IEA, Energy Technology Perspectives 2016: Towards Sustainable Urban Energy Systems, Paris: International Energy Agency, 2016, 418 pages.

[2] M. Obersteiner, C. Azar, P. Kauppi, K. Möllersten, J. Moreira, S. Nilsson, Managing Climate Risk. Science 294 (2001) 786-787.

[3] C. Azar, K. Lindgren, E. Larson, K. Möllersten, Carbon capture and storage from fossil fuels and biomass costs and potential role in stabilizing the atmosphère, Climatic Change 74 (2006) 47-49.

[4] C. Azar, K. Lindgren, M. Obersteiner, K. Riahi, D. Van Vuuren, K. Michel, The feasibility of low $\mathrm{CO}_{2}$ concentration targets and the role of bio-energy with carbon capture and storage (BECCS), Climatic Change 100 (2010) 195-202.

[5] R. Katofsky, M. Stanberry, L. Frantzis, Achieving climate stabilization in an insecure world: does renewable energy hold the key? Report International Energy Agency - Renewable Energy Technology Development, 2010.

[6] P. Luckow, M. Wise, J.J. Dooley, S. Kim, Large-scale utilization of biomass energy and carbon dioxide capture and storage in the transport and electricity sectors under stringent $\mathrm{CO}_{2}$ concentration limit scenario, International Journal of Greenhouse Gas Control 4 (2010) 865-877.

[7] O. Ricci and S. Selosse, Global and regional potential for bioelectricity with carbon capture and storage, Energy Policy 52 (2013) 689-698.

[8] M. van den Broek, V. Veenendaal, P. Koutstaal, W. Turkenburg, A. Faaij, Impact of international climate policies on $\mathrm{CO}_{2}$ capture and storage deployment: Illustrated in the Dutch energy system, Energy Policy 39 (2011) 2000-2019.

[9] D. van Vuuren, M. Den Elzen, P. Lucas, B. Eickhout, B. Strengers, Stabilizing greenhouse gas concentrations at low levels: an assessment of reduction strategies and costs, Climatic Change 81 (2007) 119-159.

[10] D. van Vuuren, J. van Vliet, E. Stehfest, Future bio-energy potential under various natural constraints, Energy Policy 37 (2009) 4220-4230.

[11] K. Tokimatsu, R. Yasuoka and M. Nishio, Global zero emission scenarios: The role of biomass energy with carbon capture and storage by forested land use, Applied Energy 185 (2017) 1899-1906.

[12] R.B. Jackson, J.G. Canadell, S. Fuss, J. Milne, N. Nakicenovic and M. Tavoni, Focus on negative emissions, Environmental Research Letters 12 (2017) 110201.

[13] M. Muratori, K. Calvin, M. Wise, P. Kyle and J. Edmonds, Global economic consequences of deploying bioenergy with carbon capture and storage (BECCS), Environmental Research Letters 11 (2016) 095004. 
[14] S. Fuss, J.G. Canadell, G.P. Peters, M. Tavoni, R.M. Andrew, P. Ciais, R.B. Jackson, C.D. Jones, F. Kraxner, N. Nakicenovic, C. Le Quéré, M.R. Raupach, A. Sharifi, P. Smith and Y. Yamagata, Betting on negative emissions, Nature Climate Change 4 (2014) 850-853.

[15] P. Viebahn, D. Vallentin and S. Höller, Prospects of carbon capture and storage (CCS) in China's power sector - An integrated assessment, Applied Energy 157 (2015) 229-244.

[16] R. Loulou, ETSAP-TIAM: The TIMES integrated assessment model. Part II: Mathematical formulation, Computational Management Science 5 (2008) 41-66.

[17] R. Loulou and M. Labriet, ETSAP-TIAM: the TIMES integrated assessment model Part I: Model structure, Computational Management Science, Special issue Managing Energy and the Environment, 5(1) (2008) 7-40.

[18] C. Hendriks, W. Graus, F. van Bergen, Global carbon dioxide storage potential and costs. Report by Ecofys in cooperation with TNO. EEP-02001 by order of the: Rijksinstituut voor Volksgezondheid en Milieu (Dutch National Institute for Public Health and the Environment, 2004.

[19] J.J. Dooley, S.H. Kim, J.A. Edmonds, S.J. Friedman and M.A. Wise, A First Order Global Geologic $\mathrm{CO}_{2}$ Storage Potential Supply Curve and its Application in a Global Integrated Assessment Model, Greenhouse Gas Control Technologies Volume I (2005) eds ES Rubin, DW Keith, and CF Gilboy, Elsevier Science, 573-581.

[20] J.J. Dooley and S.J. Friedman, A Global but Regionally Disaggregated Accounting of $\mathrm{CO}_{2}$ Storage Capacity: Data and Assumptions for Compiling Regional $\mathrm{CO}_{2}$ Storage Capacity Supply Curves for Incorporation within ObjECTS>MiniCAM., Lawrence Livermore National Laboratory. 2005, UCRL-SR-209663.

[21] U.S. DOE/NETL, Carbon Utilization and Storage Atlas - Fourth Edition (Atlas IV), 2012, 130p. http://www.netl.doe.gov/research/coal/carbon-storage/natcarb-atlas

[22] NACSA, First Edition. 2012, 52p.

http://www.netl.doe.gov/technologies/carbon_seq/refshelf/NACSA2012.pdf

[23] S. Selosse and O. Ricci, Carbon capture and storage: Lessons from a storage potential and localization analysis, Applied Energy, Volume 188, 15 (2017) 32-44.

[24] R. Wright, F. Mourits, L. Beltran Rodriguez, M. Davila Serrano, The first North American Carbon Storage Atlas, Energy Procedia, Volume 37 (2013) 5280-5289.

[25] S. Bachu, D. Bonijoly, J. Bradshaw, R. Burruss, S. Holloway, N-P. Christensen \& O-M. Mathiassen, $\mathrm{CO}_{2}$ storage capacity estimation: Methodology and gaps, International Journal of Greenhouse Gas Control 1 (2007) 430-443.

[26] S. Bachu, Screening and ranking of sedimentary basins for sequestration of $\mathrm{CO}_{2}$ in geological media in response to climate change, Environmental Geology 44 (2003) 277-289.

[27] J. Bradshaw, S. Bachu, D. Bonijoly, R. Burruss, S. Holloway, N-P. Christensen \& O-M. Mathiassen, $\mathrm{CO}_{2}$ storage capacity estimation: Issues and developments of standards, International Journal of Greenhouse Gas Control 1 (2007) 62-68.

[28] J.J. Dooley, Carbon dioxide capture and geologic storage: a core element if a global energy technology strategy to address climate change, A technology report from the second phase of the global energy technology strategy program, 2006, April, 37p. 
[29] R.T. Dahowski, C.L. Davidson, J.J. Dooley, Comparing large scale CCS deployment potential in the USA and China: a detailed analysis based on country-specific $\mathrm{CO}_{2}$ transport \& storage cost curves, Energy Procedia 4 (2011) 2732-2739.

[30] K. Michael, M. Arnot, P. Cook, J. Ennis-King, R. Funnell, J. Kaldi, D. Kirste and L. Paterson, $\mathrm{CO}_{2}$ storage in saline aquifers I - current state of scientific knowledge, Energy Procedia, Volume 1, Issue 1(2009) 3197-3204.

[31] K. Michael, A. Allinson, A. Golab, S. Sharma and V. Shulakova, $\mathrm{CO}_{2}$ storage in saline aquifers II - experience from existing storage operations", Energy Procedia, Volume 1, Issue 1 (2009) 19731980.

[32] I. Kolenkovic, B. Saftic and D. Peresin, Regional capacity estimates for $\mathrm{CO}_{2}$ geological storage in deep saline aquifers - Upper Miocene sandstones in the SW part of the Pannonian basin, International Journal of Greenhouse Gas Control 16 (2013) 180-186.

[33] M. van den Broek, E. Brederode, A. Ramirez, L. Kramers, M. van der Kuip, T. Wildenborg, W. Turkenburg and A. Faaij, Designing a cost-effective $\mathrm{CO}_{2}$ storage infrastructure using a GIS based linear optimization energy model, Environmental Modelling and Software 25 (2010) 1754-1768.

[34] H. Haberl, T. Beringer, S.C. Bhattacharya, K.H. Erb, M. Hoogwijk, The global technical potential of bio-energy in 2050 considering sustainability constraints, Current Opinion in Environmental Sustainability 2(5-6) (2010) $394-403$.

[35] IPCC (Intergovernmental Panel on Climate Change), 2011. Renewables Energy Sources and Climate Change Mitigation, Special Report, Chapter 2. Cambridge University Press, Cambridge.

[36] V. Dornburg, A. Faaij, P.A. Verweij, H. Langeveld, G. van de Ven, F. Wester, Biomass assessment: assessment of global biomass potentials and their links to food, water, biodiversity, energy demand and economy. Climate change scientific assessment and policy analysis (WAB) programme, 2008.

[37] S. Kang, S. Selosse, N. Maïzi, Contribution of global GHG reduction pledges to bioenergy expansion, Journal of Biomass \& Bioenergy, Volume 111 (2018) 142-153.

[38] S. Kang, La place de la bioénergie dans un monde sobre en carbone : Analyse prospective et développement de la filière biomasse dans le modèle TIAM-FR. Thèse de doctorat MINES ParisTech PSL Research University, 2017, Co-directed by N. Maïzi and S. Selosse,

[39] S. Kang, S. Selosse, N. Maïzi, Is GHG mitigation policy enough to develop bioenergy in Asia: a long-term analysis with TIAM-FR, International Journal of Oil, Gas and Coal Technology (IJOGCT), Special Issue on: IBSCE 2015 Bioenergy and Biofuels in Asia and Europe, 14 (1/2), (2017) 5-31.

[40] S. Kang, S. Selosse, N. Maïzi, Strategy of bioenergy development in the largest energy consumers of Asia (China, India, Japan and South Korea), Energy Strategy Reviews 8 (2015) 56-65.

[41] E. Smeets, A. Faaij, I. Lewandowski, A quickscan of global bio-energy potentials to 2050 An analysis of the regional availability of biomass resources for export in relation to the underlying factors, Bio-EnergyTrade(2004) nMarch, 0-121.

[42] IIASA/FAO. Global Agro-ecological Zones (GAEZ v3.0) [En ligne]. 2012. p. 179. Available on : http://www.fao.org/nr/gaez/en/ 
[43] FAO. « AQUASTAT ». [s.l.] : [s.n.], 2016. Available on :

http://www.fao.org/nr/water/aquastat/tables/index.stm

[44] Eurostat. « Economy-wide Material Flow Accounts (EW-MFA). Compilation Guide 2012 » 2012. nJuly, p. 68.

[45] F. Krausmann, K-H. Erb, S. Gingrich, C. Lauk, H. Haberl, Global patterns of socioeconomic biomass flows in the year 2000: A comprehensive assessment of supply, consumption and constraints, Ecological Economics, Volume 65, n³, (2008) 471-487.

[46] D. Jölli, S. Giljum, Unused biomass extraction in agriculture , forestry and fishery, 2005. Data SERI Studies $n^{\circ} 3$.

[47] G. Fischer, E. Hizsnyik, S. Prieler, H. van Velthuizen, Assessment of biomass potentials for biofuel feedstock production in Europe : Methodology and results, 2007, nºJuly, p. 75.

[48] M. Hoogwijk, On the global and regional potential of renewable energy sources. 2004. Utrecht University. Thesis. 256 p. ISBN 90-393-3640-7

[49] H.-H Rogner, R. F. Aguilera, C. Archer, R. Bertani, S. C. Bhattacharya, M. B. Dusseault, L. Gagnon, H. Haberl, M. Hoogwijk, A. Johnson, M.L. Rogner, H. Wagner, V. Yakushev, A. Faaij, Energy resources and potentials, In: Global Energy Assessment-Toward a Sustainable Future. Laxenburg, Austria: International Institute for Applied Systems Analysis, 2012. p. 423-512.

[50] E. M. W. Smeets, A. Faaij, Bioenergy potentials from forestry in 2050: An assessment of the drivers that determine the potentials, Climatic Change, Volume 81 (2007) 353-390.

[51] H. Yamamoto, K. Yamaji, J. Fujino, Evaluation of bioenergy resources with a global land use and energy model formulated with SD technique, Applied Energy, Volume 63 (1999) 101-113.

[52] E. M. W. Smeets, A. Faaij, I.M. Lewandowski, W.C. Turkenburg, A bottom-up assessment and review of global bio-energy potentials to 2050, Progress in Energy and Combustion Science [online], Volume 33 (2007) 56-106.

[53] D. Bodansky, The Paris Climate Change Agreement: a new hope?, American Journal of International Law, Volume 110, No. 2 (2016) 288-319.

[54] M. Luomi, Is the Paris Agreement a success and what does it mean for the energy sector?, Oxford Energy Forum, Issue 105, (2016) 4-7.

\section{Acronyms list}

AR: Assessment report

BECCS: Bioenergy with carbon capture and storage

$\mathrm{CH}_{4}$ : Methane

$\mathrm{CO}_{2}$ : Carbon dioxide

COP: Conference of Parties

CCS: Carbon capture and storage

DM: Dry matter

E3MG: Energy-Environment-Economy Model at the Global level

EJ: Exajoule

EOR: Enhanced oil recovery 
ETSAP: Energy Technology Systems Analysis Program

FAO: Food and Agriculture Organization

GAEZ: Global Agro-ecological Zones

GAMS: General Algebraic Modeling System

GHG: Greenhouse gas

Gt: Gigaton

GW: Gigawatt

$\mathrm{H}_{2}$ : Hydrogen

IAM: Integrated Assessment Model

IEA: International Energy Agency

IPCC: Intergovernmental Panel on Climate Change

MERGE: Model for Evaluating the Regional and Global Effects of GHG Reduction Policies

$\mathrm{N}_{2} \mathrm{O}$ : Nitrous oxide

NE: Negative emissions

$\mathrm{O}_{2}$ : Dioxygen

OECD: Organisation for Economic Co-operation and Development

PJ: Petajoule

POLES: Prospective Outlook on Long-term Energy Systems

REMIND: Regionalized Model of Investments and Development

RES: Reference Energy System

TIAM: TIMES Integrated Assessment Model

TIMER: Targets IMage Energy Regional

TOF: Tree outside the forest

UNFCCC: United Nations Framework Convention on Climate Change 